Journal for ImmunoTherapy of Cancer Thomas G, Salek-Ardakani S. CXCL9-expressing tumorassociated macrophages: new players in the fight against cancer. Journal for ImmunoTherapy of Cancer 2021;9:e002045. doi:10.1136/ jitc-2020-002045

Accepted 28 December 2020

Check for updates

(c) Author(s) (or their employer(s)) 2021. Re-use permitted under CC BY-NC. No commercial re-use. See rights and permissions. Published by BMJ.

Cancer Immunology Discovery, Pfizer Inc, San Diego, California, USA

\section{Correspondence to} Dr Shahram Salek-Ardakani; shahram.salek-ardakani@pfizer. com

\title{
CXCL9-expressing tumor-associated macrophages: new players in the fight against cancer
}

\author{
Paola Marie Marcovecchio, Graham Thomas, Shahram Salek-Ardakani
}

\begin{abstract}
Tumor-associated macrophages (TAMs) are among the main contributors to immune suppression in the tumor microenvironment, however, TAM depletion strategies have yielded little clinical benefit. Here, we discuss the concept that TAMs are also key regulators of anti-PD(L)1-mediated CD8 T cell-dependent immunity. Emerging data suggest that expression of the chemokine CXCL9 by TAMs regulates the recruitment and positioning of CXCR3-expressing stem-like CD8 $\mathrm{T}\left(\mathrm{T}_{\text {stem }}\right)$ cells that underlie clinical responses to anti-PD(L)-1 treatment. We evaluate clinical and mechanistic studies that establish relationships between CXCL9-expressing TAMs, $T_{\text {stem }}$ and antitumor immunity. Therapies that enhance anti-PD(L)-1 response rates must consider TAM CXCL9 expression. In this perspective, we discuss opportunities to enhance the frequency and function of CXCL9 expressing TAMs and draw on comparative analyzes from infectious disease models to highlight potential functions of these cells beyond $\mathrm{T}_{\text {stem }}$ recruitment.
\end{abstract}

\section{INTRODUCTION}

In the past decade, checkpoint blockade therapies targeting programmed death 1 or programmed death ligand 1 (PD1/PD-L1; PD1/PD-L1, referred to as anti-PD(L)-1) have moved immunotherapy into the forefront of clinical care and have transformed our understanding of tumor-immune interactions. Although anti-PD(L)-1 therapy works exceedingly well in approximately $30 \%$ of patients, failure in the remaining $70 \%$ has driven intense efforts to understand the cellular and molecular pathways underlying effective treatment. ${ }^{1}$ PD1 antagonists target inhibitory PD1 receptor signaling on cytotoxic $\mathrm{T}$ lymphocytes to enhance cancer cell killing. ${ }^{2}$ Effective responses are understood to require cytotoxic CD8 T cells recruitment into the tumor to exert their cytolytic effects. Within the tumor microenvironment (TME), sustained and robust antitumor responses are impaired by processes such as immunoediting, loss of antigen expression, $\mathrm{T}$ cell exclusion, the presence of a highly immunosuppressive milieu and $\mathrm{T}$ cell exhaustion and death. ${ }^{3}{ }^{4}$ Therefore, maximizing $\mathrm{T}$ cell recruitment to the tumor and preventing $\mathrm{T}$ cell dysfunction within the TME are two fundamental parameters that may be influenced further to enhance response rates to anti-PD(L)-1 therapy. ${ }^{5}$

Myeloid cells are a significant component of the TME and are predominantly immunosuppressive, constituting a major source of resistance to anti-PD(L)-1 efficacy. ${ }^{6}$ The c-Fms tyrosine kinase receptor (colony-stimulating factor 1, CSF-1R) is essential for developing and maintaining monocyte and macrophage lineage cells. CSF-1R inhibition has been considered an attractive, broad approach for depleting tumor-associated macrophages (TAMs) to restore CD8 T cell function within tumors. However, despite the reasoning that monocytes and TAMs contribute to immunosuppression in the $\mathrm{TME}^{7}$ clinical trials with CSF-1R inhibitors have yet to show significant benefit, except for diffuse-type giant cell tumors. ${ }^{89}$ As macrophages and their precursors are often the predominant immune cell type present inside and around the TME, the cues that these cells provide and their interactions with $\mathrm{T}$ cells play a critical role in shaping the outcome of anti-PD(L)-1 treatment.

A growing body of evidence points to the CXCR3 chemokine pathway as a significant axis of anti-PD(L)-1 therapy response, regulating the recruitment and positioning of effector $\mathrm{T}$ cells within the TME. ${ }^{10-12}$ Interferon (IFN)-induced CXCR3 ligands, CXCL9/10/11, regulate tumor angiogenesis, ${ }^{13}$ enhance $\mathrm{T}$ cell infiltration and further position activated $\mathrm{T}$ cells near antigenpresenting cells within the TME, which may provide additional queues to $\mathrm{T}$ cells that facilitate antitumor immunity. As myeloid cells are major producers of CXCR3 ligands, one potential explanation for the lack of efficacy with CSF1R inhibitors is the unintended consequence of depleting myeloid populations that support antitumor immunity, for instance, via the production of CXCL9. 
Therefore, the therapeutic challenge appears to be retaining or even enhancing, myeloid-derived CXCR3 ligand production and functionality within the TME while overcoming the immune-suppressive barriers imposed by myeloid cells.

In this review, we discuss recent evidence supporting the role played by TAMs in facilitating anti-PD(L)-1 responses by recruiting or positioning functional CD8 $\mathrm{T}$ cells. We will examine intratumoral myeloid heterogeneity and discuss the phenotype of antitumor myeloid populations as recently revealed using high-dimensional phenotyping approaches. In light of these recent efforts to more carefully and thoroughly characterize the diversity and function of TAMs in various tumors, we consider the implications of this emerging myeloid biology for antitumor CD8 $\mathrm{T}$ cell responses and discuss potential opportunities to enhance anti-PD(L)-1 responses that account for these considerations.

\section{Heterogeneity of macrophages and their precursors implies antitumoral phenotypes}

Early paradigms of macrophage polarization in cancer centered around the perceived dichotomy between inflammatory 'M1' and suppressive 'M2' subsets based on similarities in surface marker expression. These paradigms were borrowed from in vitro models of activation using TLR agonists such as lipopolysaccharide (LPS) for $\mathrm{M} 1$, or cytokines such as IL-4 for M2. ${ }^{14}$ It is now appreciated that the tumor macrophage compartment exists as a heterogeneous admixture of phenotypes that arise from cells of embryonic or bone-marrow monocyte origins, whose differentiation state is determined by cellular developmental history, residency time within the tumor and the environmental cues imparted by the anatomical niche. ${ }^{15-20}$

High-resolution analyzes of TAM heterogeneity have affirmed the negative associations between TAM abundance and survival, ${ }^{17} 18$ emphasizing these cells' capacity to suppress antitumor immunity, induce immunosuppressive cancer-associated fibroblast niches and coordinate tumor neovascularization to maintain a non-inflamed cancer state. Similar high-dimensional analyzes have also implicated MHCII ${ }^{\text {high }}$ or HLA-DR ${ }^{\text {high }}$ expressing monocytes required for effective anti-PD(L)-1 treatment. ${ }^{21}$ Specifically, CyTOF analysis of melanoma patients found that a circulating inflammatory monocyte phenotype (CD14+CD33+HLA DR ${ }^{\text {hi }}$ ICAM-1 +CD64+CD141+CD86+CD11c+CD38+PD-L1+CD11b+) correlated with response to anti-PD1 therapy. ${ }^{21}$ Furthermore, an independent study assessing peripheral monocyte sensitivity to IFN $\gamma$ stimulation was strongly associated with survival in patients with breast cancer. ${ }^{23}$

These observations and the long-held understanding that tumor-associated 'M1' macrophages are associated with a favorable prognosis raises the question as to whether these cells may play a causal role in antitumor immunity, rather than correlate with response to treatment. Such a relationship may explain the underwhelming performance of CSF1R inhibitors in the clinic, as alongside depletion of immunosuppressive TAMs, there may additionally be a loss of antitumor subsets.

\section{The CXCL9-CXCR3 axis mediates responses to anti-PD(L)-1 therapy}

Dangaj et al recently published an analysis of TCGA datasets that aimed to identify chemokines correlating with CD8a gene expression across various tumor indications including kidney, lung, colon, breast, ovarian and uterine cancers. ${ }^{11}$ Here, the authors found a strong positive correlation between CD8a and CXCL9 and, to a lesser extent, CXCL10 and CXCL11, gene expression. These chemokines are all ligands for the CXCR3 receptor, expressed on activated CD8 T cells and natural killer (NK) cells. ${ }^{24}$ To explore the potential sources of CXCL9 in the TME, the authors performed immunofluorescence staining in epithelial ovarian cancer, showing colocalization of CXCL9 with CD68 positive macrophages and CD11c+ dendritic cells (DCs). Tumors with absent stromal CXCL9 expression were found to contain CD8 T cells restricted to the tumor margin. In the presence of CXCL9 expression, CD8+ T cells were frequently observed infiltrating the tumor islets. ${ }^{11}$ These findings establish a relationship between CXCL9 expression and intratumoral CD8 $\mathrm{T}$ cell positioning. Work by others, including our group ${ }^{1225}$ have further established a relationship between tumor CXCL9 gene expression and survival with clinical anti-PD(L)-1 inhibitors avelumab and atezolizumab in multiple indications, including melanoma and metastatic urothelial carcinoma. ${ }^{125}$

A combination of antibody blocking and genetic approaches have been used to demonstrate the role of the CXCL9-CXCR3 axis in response to anti-PD(L)-1 therapy and to delineate cellular contributions. Building on earlier studies describing a requirement for the CXCL9-CXCR3 axis in cancer, ${ }^{13}{ }^{26}$ Chow et al provided the first genetic evidence that $\mathrm{Cxcr} 3$ deficiency impairs the efficacy of anti-PD1 treatment using MC38 colorectal carcinoma. ${ }^{10}$ Cxcr3-deficient mice possess substantially increased tumor volumes compared with wild type (WT) controls following the PD1 blockade. Our group has also recently shown that blocking CXCR3 and CXCL9 impair anti-PD-L1 efficacy in CT26 colorectal carcinoma, ${ }^{25}$ while CXCR3 inhibition impairs the efficacy of dual anti-PD1 and anti-cytotoxic $\mathrm{T}$ lymphocytes antigen-4 (CTLA-4) regimen in multiple tumor models. ${ }^{12}$

Disruption of the CXCL9-CXCR3 axis is associated with reduced infiltration of peripheral CD8 $\mathrm{T}$ cells into the $\mathrm{TME}^{10} 27$ (figure 1A), while PD1 blockade increases tumor mRNA expression and circulating protein levels of CXCL9 and CXCL10. ${ }^{10}{ }^{12}$ Although both chemokines are increased, CXCL9 levels are considerably higher than CXCL10. Subsequent knock-out studies show that the genetic deletion of CXCL10 has less impact than CXCL9 deletion on tumor growth in MC38. ${ }^{10}$ The relatively higher expression of CXCL9 as opposed to CXCL10 and CXCL11 in both human and mouse models may 


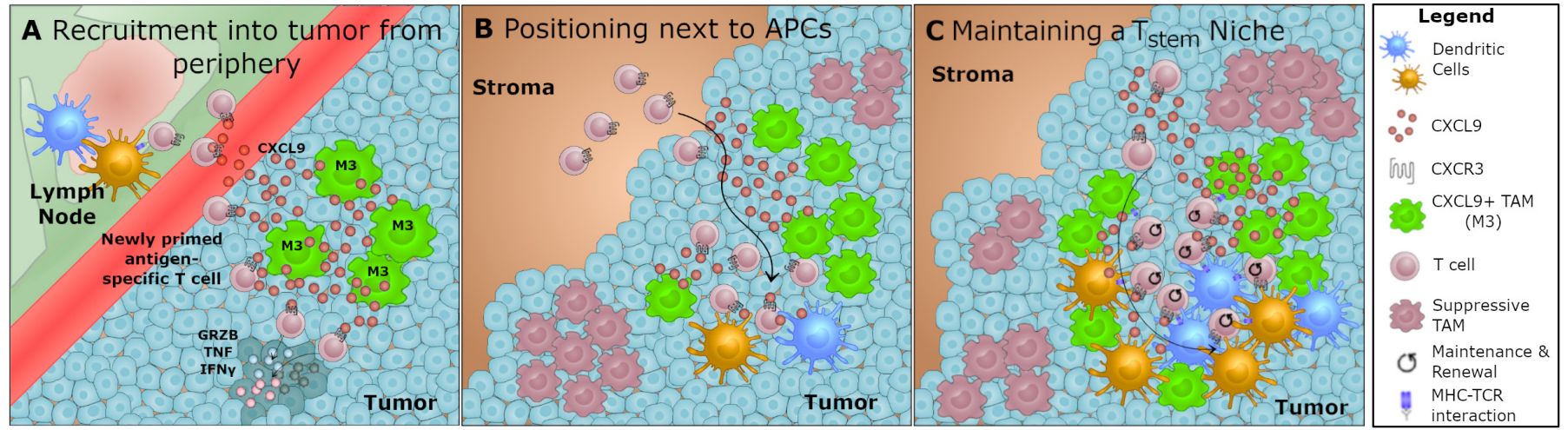

Figure 1 Scenarios of CXCL9 function in the tumor microenvironment. Expression of chemokine CXCL9 by macrophages within the tumor microenvironment may serve multiple purposes in generating an efficacious immune response to PD-L(1) checkpoint therapy. (A) Newly primed T cells in tumor draining lymph nodes upregulate CXCR3 and may be recruited to sources of CXCL9 in the tumor, allowing for infiltration of non-exhausted effector T cells. (B) CXCL9 secretion by macrophages may position newly primed effector T cells closer to APCs such as classical DCs, which have been shown to be necessary for efficacy of PD-L(1) treatment. (C) As a potential mechanism for maintaining a non-exhausted effector T cell pool, CXCL9 may be used to recruit and position TCF1+ T cells within specialized niches of the TME. APCs, antigen-presenting cells; DCs, dendritic cells; IFN, interferon; PD-L1, programmed death ligand 1; TAM, tumor-associated macrophage; TME, tumor microenvironment.

explain the apparent dominance of CXCL9 in regulating anti-PD1 responsiveness. ${ }^{11}$ IFN $\gamma$ response signatures are strongly associated with anti-PD1 efficacy ${ }^{13}$ and CXCL9 is more sensitive to induction by IFN $\gamma$ than CXCL10/11, which are more potently regulated by the type $1 \mathrm{IFN} \alpha / \beta{ }^{28}$ Notably, CXCL10 can contribute to antitumor immunity via similar mechanisms as CXCL9, as inhibition of the secreted protease DPP4 enhances antitumor immunity in a CXCL10-dependent fashion. ${ }^{29}$ DPP4 cleaves and inactivates CXCL10 in mouse but not CXCL9, adding an additional layer to the negative regulation of CXCL10 in the TME.

Blockade of the CXCL9-CXCR3 axis leads to a considerable reduction in the infiltration of CD8+ $\mathrm{T}$ cells into the TME following anti-PD1 treatment in both the AT3 breast and MC38 colorectal carcinoma models. ${ }^{10}$ At the same time, mixed bone marrow chimeras between $\mathrm{WT}$ and $\mathrm{Cxcr}^{-/-}$bone marrow show significantly higher infiltration of WT CD8+ T cells into the tumor following anti-PD1 treatment. ${ }^{10}$ Thus, the CXCL9-CXCR3 axis is essential for the efficacy of anti-PD1 responses.

\section{Phenotype and regulation of CXCL9/10 expressing TAMs in the TME}

Numerous studies have addressed the source(s) of CXCL9 within the tumor, and it seems that this chemokine is predominantly derived from CD68 + macrophages and CD11c+ DCs. ${ }^{10-12252730}$ Within the TME, however, macrophages are both far more abundant than conventional DCs (cDCs) and produce higher levels of CXCL9 when measured as a frequency of total positive cells. ${ }^{1012}$ As CXCL9 expression correlates with a survival advantage, these findings indicate that TAM-derived CXCL9 is an important component of antitumor immunity. It has also been suggested that intratumoral cDCs are an important source of CXCL9, ${ }^{10}$ and cDC-derived CXCL9 may act in concert or synergy with CXCL9-expressing TAMs either within the tumor or within the draining lymph node. However, intratumoral CD11b+DCs profiled by Chow et al were found to be the major source of CXCL9/10 expression within the tumor. The FACS gating used by Chow et al however does not exclude TAMs expressing these markers by negatively gating macrophage-specific markers such as CD64(figure 1b). These observations support the concept that broad TAM depletion may remove both immune-suppressive populations and antitumor populations. Indeed, House et al showed using F4/80 depleting antibodies that loss of TAMs ablates antiPD1 and anti-CTLA-4 induced CXCL9, reversing immunotherapy efficacy. However, given the broad depleting effects of anti-F4/80 on TAM populations, such treatment is expected to provide little information on the specific phenotype of any antitumor TAM subset.

Our group recently employed a bilateral tumor model to understand immune correlates of response to avelumab (anti-PD-L1) treatment. ${ }^{25}$ By implanting CT26 colorectal carcinoma subcutaneously into both flanks, we used single-cell RNA-Seq to profile the TME before treatment with Avelumab while tracking treatment response in the contralateral tumor. Of the 26 immune cell populations identified in the TME, only a single subset correlated positively with the response to avelumab. Transcriptomic phenotyping identified an inflammatory myeloid subset, which we termed 'M3' (it was the third myeloid cluster in our dataset). This M3 population was distinct from cDC subsets, monocytes and mature macrophages. ${ }^{25}$ However, it appeared to be monocyte-derived based on single-cell trajectory analysis. Notably, M3 TAMs expressed uniquely high levels of Cxcl9, alongside MHCII, Cd40, Cd274 (PD-L1), Stat1, Nos2 and Cd38 as well as intermediate levels of Itgax (CD11c). To address whether this murine population possesses a human counterpart, we analyzed scRNA-Seq profiling data of human myeloid subsets. ${ }^{31}$ 
One population, termed Mac_9, was identified that bore a significant similarity to the murine M3 subset. Mac_9 cells shared an inflammatory gene signature with M3 TAMs, with both coexpressing multiple genes, including Cxcl9, Cxcl10, MHCII, Cd40 and Stat1. ${ }^{25}$ A recent transcriptomic profiling study of TAMs from lung cancer patients also identified variable expression of inflammatorymacrophage-associated genes, most notably Cxc19, Cxcl10 and Stat1 ascribed to an 'M1 ${ }^{\text {hot }}$ phenotype. ${ }^{30}$ Genes associated with this $\mathrm{M} 1^{\text {hot }}$ state, including Cxc19, correlated with increased survival and a higher density of intratumorous CD8 $\mathrm{T}$ cells indicating that human myeloid populations bearing a highly similar transcriptional state to murine Cxcl9 expressing-TAMs correlate with survival and accumulation of CD8 T cells in human cancer.

IFN $\gamma$, a master regulator of CXCL9 gene expression, ${ }^{24}$ is also critical for the M3 phenotype. We observed IFN $\gamma$ and Nf-kB gene signature enrichment in M3 TAMs, perhaps indicating that, in addition to IFN $\gamma$, other pathways contribute to the polarization of this subtype. ${ }^{25}$ Additionally, Ifng, but not Ifna or Ifnb, gene expression was correlated with Cxc19 expression across multiple TCGA indications. ${ }^{11}$ Furthermore, depletion of IFN $\gamma$ but not IFN $\alpha / \beta$ in human tumor-immune cell cocultures ${ }^{11}$ and in AT-3ova tumor-bearing mice substantially reduced CXCL9 levels. ${ }^{12}$

What sources of IFN $\gamma$ elicit this 'M3' phenotype? Dangaj et al found that CD8a mRNA levels also correlated with CCL5. Significantly, expression levels of CXCL9 predicted survival, while CCL5 was found to be required for CXCL9 expression. Using in vitro and in vivo models of epithelial ovarian cancer, the authors surmise that tumor-derived CCL5 recruits existing tumor-reactive T cells to overcome immune desertification. ${ }^{11}$ Upon antigen encounter, these cells produce IFN $\gamma$ to amplify antitumor immunity via myeloid-derived CXCL9. It will be essential to understand why CD8 T cells recruited via CCL5 are not sufficient to mediate tumor control, and what additional benefits are brought by the recruitment of CXCR3+CD8 T cells. Whether other cell types may provide signals to elicit the M3 TAM phenotype should also be addressed. Bilateral models used to identify predictors of response to dual anti PD1 and anti-CTLA-4 treatment identified a STAT1-NK cell axis, potentially implicating NK cell-derived IFN $\gamma$ in this process. ${ }^{32}$ Other regulators of CD8 T cell-dependent antitumor immunity have been described, for instance, NKT cells in hepatocellular carcinoma. ${ }^{33}$ The extent to which these mediators of tissue-specific immunity orchestrate their effects via IFN $\gamma$-dependent myeloid reprogramming also remains in question.

\section{CXCL9 expressing TAMs in stem-like CD8 T cell recruitment and positioning}

The studies discussed above have established a critical role for the macrophage CXCL9-CXCR3 axis in mediating responses to anti-PD1 treatment. It is informative to consider these findings in the context of how anti-PD $(\mathrm{L})-1$ influences CD8 $\mathrm{T}$ cell function to control cancer. It was once assumed that anti-PD1 blocked inhibitory signaling within exhausted CD8 T cells to derepress their function in the TME. ${ }^{345}$ These exhausted cells express high levels of inhibitory receptors, including PD1, Tim3, Lag3 and Tigit, which have limited self-renewal capacity and do not express the full repertoire of effector cytokines including IL-2 and tumor necrosis factor (TNF) $\alpha{ }^{5}$ Clonal TCR profiling experiments in humans and mice have shown that efficacious anti-PD(L)-1 treatment precipitates a turnover of the tumor TCR repertoire, demonstrating that exhausted cells within the tumor are replaced on treatment. ${ }^{56}$ The identification of a progenitor-exhausted CD8 phenotype that expresses intermediate levels of PD1, the transcription factor T cell factor 1 (TCF1, Tcf7), and expands in response to anti-PD1 treatment has unveiled the right cellular target of action of anti-PD (L)-1 therapies. ${ }^{19}{ }^{37}$ Higher frequencies of TCF $1+$ progenitorexhausted CD8 T cells within human tumors is a favorable prognostic indicator. ${ }^{38}$ Using CXCR3GFP (CIBER) reporter mice treated with anti-PD1, Chow et al show that CXCR3+ CD8 T cells include progenitor-exhausted phenotype cells expressing lower levels of Tim3, Lag3 and PD1, in contrast to activated CXCR3 ${ }^{-/}$CD8 cells. Furthermore, competitive mixed bone marrow chimera studies using WT and $\mathrm{Cxcr3}^{-/-}$bone marrow showed that WT CD8 T cells produced higher effector cytokines, measured as increased IFN $\gamma+\mathrm{TNF} \alpha+$ double-positive cells following re-stimulation. ${ }^{10}$ Thus, the recruitment of CXCR3+ progenitor-exhausted CD8 T cells via CXCL9 dependent mechanisms is a critical step in response to immune checkpoint blockade.

TCF1+CD8 T cells have recently been shown to occupy 'niches' within the tumor composed of dense MHCII+ cell clusters. ${ }^{19}$ These TCF1 + cells are proposed to give rise to highly proliferative effector subsets. ${ }^{19}$ Such niches resemble the $\mathrm{T}$ cell zone of secondary lymphatics, but the extent to which CXCL9 is secreted, or how these niches facilitate TCF1 +CD8 T cell abundance within the TME is yet to be addressed. One interpretation of the niche hypothesis is that these MHCII +regions do not represent stable microenvironments for $\mathrm{TCF} 1+\mathrm{CD} 8+\mathrm{T}$ cell persistence (figure 1C). As histological images of MHCII+ and CD8+TCF1+ cell co-localization represent a snapshot, these observations may simply provide information on the positioning of recently recruited CXCR3 +CD8 T cells infiltrating the TME from the periphery. Alternatively, these MHCII+ cell niches may support intratumor $\mathrm{TCF} 1+\mathrm{CD} 8+\mathrm{T}$ cell longevity through as yet uncharacterized mechanisms. ${ }^{39}{ }^{40}$ Irrespective of their precise role, loss of these MHCII+ niches within human tumors was associated with impaired CD8 T cell responses, and disease progression. ${ }^{19}$ An improved understanding of how these niches form, including a catalog of their cellular constituents, and mapping relationships between niche subsets may yield novel therapeutic opportunities to enhance M3 TAM differentiation and antitumor immunity. 


\section{Additional roles beyond recruitment?}

Whether M3 TAMs play additional roles beyond recruiting CXCR3+ T cells remains an open question. Based on scRNA-Seq profiling, we observe that the M3 phenotype bears similarity to $\mathrm{TNF} \alpha / \mathrm{iNos}$ producing DCs (TipDC) in both ontogeny and marker expression. ${ }^{41}$ Predominantly discussed in the context of infectious disease, TipDCs are monocyte-derived and are $\mathrm{CD} 11 \mathrm{~b}^{\text {int }} \mathrm{Cd} 11 \mathrm{c}^{\text {int }} \mathrm{Cd} 40+\mathrm{M}-$ HCII+ by flow cytometry, consistent with the M3 gene expression signature. ${ }^{41}{ }^{42}$ While dispensable for lymph node priming of CD8 $\mathrm{T}$ cell responses ${ }^{41}$ TipDCs effectively amplify antigen-specific responses within infected tissues. ${ }^{42}$ Respiratory virus infection models have shown that lung monocyte-derived cells support the generation of lung CD8 T cell tissue-resident memory (Trm) populations $^{43}$ in a manner that depends on engagement of costimulatory receptors including GITR, ${ }^{44}$ and perhaps LIGHT $^{45}$ and HVEM $^{46}$ expressed on T cells within the tissue. Few studies have evaluated the role(s) of TipDCs in cancer. However, it has been shown that Nos2 expression in $\mathrm{CD} 11 \mathrm{~b}^{\text {int }} \mathrm{CD} 11 \mathrm{c}^{\text {int }}$ cells is important for effective control of tumor growth mediated by transferred ovalbuminspecific OT-1 T cells and that this is augmented by CD40 agonist treatment. ${ }^{47}$ This implies that activated myeloid populations in the tumor with M3 TAM-like properties may serve a TipDC-like function. Interestingly, enrichment of a CD8 Trm signature was found in NSCLC patient samples containing higher levels of CXCL9 when compared with CXCL9 negative tumors. ${ }^{30}$ This study suggested that M1 ${ }^{\text {hot }}$ TAMs provide fatty acids to CD8+CD103+Trm cells within the TME, a known mechanism for supporting peripheral CD8 T cell effector functions. ${ }^{48}$ Further, CXCL9/10 expressing macrophages have been shown to directly promote effector CD8 T cell function. In the lymph node, antigen-experienced effector memory CD8 T cells localize to the subcapsular sinus as a result of macrophage CXCL9/10 expression, ${ }^{49}$ this enables efficient interaction of memory cells with cognate antigen draining to the lymphatics on reinfection. Notably, CXCR3 deficient CD8 T cells were less likely to attain a short-lived effector phenotype in a vaccinia-virus model. ${ }^{50}$ Whether CXCL9 expressing M3 TAMs regulate CD8 T cell function in the tumor by promoting longevity (ie, a Trm-type response), by enhancing effector functions, both or via other mechanisms remains to be determined. Future studies will be required to ascertain any additional roles for M3 TAMs within the TME that may influence tumor infiltrating CD8 T cell effector functions beyond recruitment via CXCL9.

Multiple cell populations in the TME express CXCL9, including DCs. ${ }^{102527}$ Indeed, some studies have concluded that $\mathrm{CDC}(\mathrm{cDC})$ populations within the TME are essential for ICB efficacy in the context of CXCL9 deletion. ${ }^{10}$ In our studies, we observe low expression of CXCL9 and CXCL10 by cDCs, plasmacytoid DCs (pDCs), and myeloid-derived CDC2 populations in the TME prior to anti-PD-L1 treatment. ${ }^{25}$ However, these chemokines are broadly induced in response to treatment ${ }^{102527}$ and so this does not exclude a role for intratumor $\mathrm{cDCs}$ or other CXCL9 expressing populations. cDCs are undoubtedly of critical importance for antitumor immunity. However, one caveat of genetic deficiency models is that they cannot discriminate between a role for CDC-derived CXCL9 within secondary lymphatics, as is known to occur, ${ }^{51}$ or at the tumor site. Furthermore, FACS and microscopy-based classification of tumor DCs based on MHCII and CD11c expression would not exclude the TipDC like M3 TAM population. ${ }^{12}{ }^{19}$ Given the strong association between tumor CXCL9 expression and overall survival in clinical data, ${ }^{11} 1225$ understanding whether all CXCL9-expressing cells within the tumor exert a similar influence on antitumor immune responses will be necessary. Indeed, some evidence exists that this is not the case. An immunosuppressive IDO1+CXCL9+TAM population has recently been associated with progressive disease in non-smallcell lung cancer. ${ }^{52}$ In this study, patients with progressive disease possessed fewer overall $\mathrm{T}$ cells, yet relatively higher frequencies of Foxp3+CD4 Tregs and Pdcd1 (PD1) positive exhausted CD8 T cells. While CXCL9 may be a critical regulator of $\mathrm{CD} 8 \mathrm{~T}$ cell infiltration and positioning within the TME, the functional consequences of CXCR3+CD8 T cell interactions with distinct CXCL9 (or CXCL10/11) expressing cells in the tumor may be quite different.

\section{CONCLUSIONS AND FUTURE PERSPECTIVES}

Future studies should address the functional consequences of interactions between CXCL9 expressing myeloid cells and CXCR3+TCF1+ T cells in the TME to determine potential consequences for downstream effector T cell activation. With respect to recruitment or positioning of CXCR3+ T cells within the TME, a greater understanding of the type of $\mathrm{T}$ cell that expresses CXCR3 that eventually enters the tumor and what its fate may be after being positioned near CXCL9 +myeloid cells remains to be investigated within the tumor.

Regarding therapeutic avenues, it is clear that broad depletion of monocyte/macrophage lineage cells may undermine the efficacy of immune checkpoint inhibition via the depletion of CXCL9-expressing TAMs, curtailing the infiltration of antitumor CD8 T cells. Viewed through this lens, promising therapeutic approaches to combine with anti-PD1 therapy include agonizing M3-like cells directly to enhance their function, for instance, using CD40 agonists. ${ }^{53-55}$ Alternatively, exploiting opportunities to increase the stability of the CXCR3-CXCL9/10 axis may prove promising, for instance, enhancing CXCL10 protein stability by DPP4 inhibition, ${ }^{29}$ or preventing the downregulation of CD8 T cell CXCR3 expression by tumor-derived TGF $\beta .^{56}$ Finally, depletion of mature macrophages may allow for enhanced infiltration of inflammatory monocytes with the potential to differentiate into CXCL9-expressing TAMs, such as has recently been shown using Lif, CD163, Trem2 or Tyro-Axl-Mer receptor antagonists. ${ }^{57-60}$ In this context, there should be 
considerable interest in understanding whether CXCL9expressing myeloid populations exhibit differential states of activation in response to inflammatory agonists, such as CD40, to determine the consequences of these on antitumorous $\mathrm{T}$ cell responses.

\section{Contributors The authors contributed equally to all aspects of this article.}

Funding The authors have not declared a specific grant for this research from any funding agency in the public, commercial or not-for-profit sectors.

Competing interests All authors are employees of Pfizer. SS-A and GT are Pfizer shareholders.

\section{Patient consent for publication Not required.}

Provenance and peer review Not commissioned; externally peer reviewed.

Open access This is an open access article distributed in accordance with the Creative Commons Attribution Non Commercial (CC BY-NC 4.0) license, which permits others to distribute, remix, adapt, build upon this work non-commercially, and license their derivative works on different terms, provided the original work is properly cited, appropriate credit is given, any changes made indicated, and the use is non-commercial. See http://creativecommons.org/licenses/by-nc/4.0/.

\section{ORCID iD}

Shahram Salek-Ardakani http://orcid.org/0000-0002-2336-8452

\section{REFERENCES}

1 Ribas A, Wolchok JD. Cancer immunotherapy using checkpoint blockade. Science 2018;359:1350-5.

2 Wei SC, Duffy CR, Allison JP. Fundamental mechanisms of immune checkpoint blockade therapy. Cancer Discov 2018;8:1069-86.

3 Hegde PS, Chen DS. Top 10 challenges in cancer immunotherapy. Immunity 2020;52:17-35.

4 DeNardo DG, Ruffell B. Macrophages as regulators of tumour immunity and immunotherapy. Nat Rev Immunol 2019;19:369-82.

5 Jiang P, Gu S, Pan D, et al. Signatures of T cell dysfunction and exclusion predict cancer immunotherapy response. Nat Med 2018;24:1550-8.

6 Gabrilovich DI, Nagaraj S. Myeloid-Derived suppressor cells as regulators of the immune system. Nat Rev Immunol 2009;9:162-74.

7 Strachan DC, Ruffell B, Oei Y, et al. CSF1R inhibition delays cervical and mammary tumor growth in murine models by attenuating the turnover of tumor-associated macrophages and enhancing infiltration by CD8 T cells. Oncoimmunology 2013;2:e26968.

8 Quail DF, Joyce JA. Molecular pathways: deciphering mechanisms of resistance to macrophage-targeted therapies. Clin Cancer Res 2017;23:876-84.

9 Cannarile MA, Weisser M, Jacob W, et al. Colony-Stimulating factor 1 receptor (CSF1R) inhibitors in cancer therapy. j. immunotherapy cancer 2017;5:53.

10 Chow MT, Ozga AJ, Servis RL, et al. Intratumoral activity of the CXCR3 chemokine system is required for the efficacy of anti-PD-1 therapy. Immunity 2019;50:1498-512.

11 Dangaj D, Bruand M, Grimm AJ, et al. Cooperation between constitutive and inducible chemokines enables $\mathrm{T}$ cell engraftment and immune attack in solid tumors. Cancer Cell 2019;35:885-900.

12 House IG, Savas P, Lai J, et al. Macrophage-Derived CXCL9 and CXCL10 are required for antitumor immune responses following immune checkpoint blockade. Clin Cancer Res 2020;26:487-504.

13 Mikucki ME, Fisher DT, Matsuzaki J, et al. Non-Redundant requirement for $\mathrm{CXCR} 3$ signalling during tumoricidal T-cell trafficking across tumour vascular checkpoints. Nat Commun 2015;6:7458.

14 Murray PJ, Allen JE, Biswas SK, et al. Macrophage activation and polarization: Nomenclature and experimental guidelines. Immunity 2014;41:14-20.

15 Lavin Y, Kobayashi S, Leader A, et al. Innate immune landscape in early lung adenocarcinoma by paired single-cell analyses. Cell 2017;169:750-65.

16 Azizi E, Carr AJ, Plitas G, et al. Single-Cell map of diverse immune phenotypes in the breast tumor microenvironment. Cell 2018;174:1293-308.

17 Chevrier S, Levine JH, Zanotelli VRT, et al. An immune atlas of clear cell renal cell carcinoma. Cell 2017;169:736-49.

18 Cassetta L, Fragkogianni S, Sims AH, et al. Human tumor-associated macrophage and monocyte transcriptional landscapes reveal cancer- specific reprogramming, biomarkers, and therapeutic targets. Cancer Cell 2019;35:588-602.

19 Jansen CS, Prokhnevska N, Master VA, et al. An intra-tumoral niche maintains and differentiates stem-like CD8 T cells. Nature 2019;576:465-70.

20 Ginhoux F, Guilliams M. Tissue-Resident macrophage ontogeny and homeostasis. Immunity 2016;44:439-49.

21 Krieg C, Nowicka M, Guglietta S, et al. High-Dimensional single-cell analysis predicts response to anti-PD-1 immunotherapy. Nat Med 2018;24:144-53.

22 Huang M-N, Nicholson LT, Batich KA, et al. Antigen-loaded monocyte administration induces potent therapeutic antitumor $\mathrm{T}$ cell responses. J Clin Invest 2020;130:774-88.

23 Wang L, Simons DL, Lu X, et al. Breast cancer induces systemic immune changes on cytokine signaling in peripheral blood monocytes and lymphocytes. EBioMedicine 2020;52:102631.

24 Tokunaga R, Zhang W, Naseem M, et al. Cxcl9, CXCL10, CXCL11/ CXCR3 axis for immune activation - a target for novel cancer therapy. Cancer Treat Rev 2018;63:40-7.

$25 \mathrm{Qu}$ Y, Wen J, Thomas G, et al. Baseline frequency of inflammatory Cxcl9-Expressing tumor-associated macrophages predicts response to Avelumab treatment. Cell Rep 2020;32:107873.

26 Andersson Åsa, Yang S-C, Huang M, et al. II-7 promotes CXCR3 ligand-dependent $\mathrm{T}$ cell antitumor reactivity in lung cancer. $J$ Immunol 2009;182:6951-8.

27 Rashidian M, LaFleur MW, Verschoor VL, et al. Immuno-PET identifies the myeloid compartment as a key contributor to the outcome of the antitumor response under PD-1 blockade. Proc Natl Acad Sci U S A 2019;116:16971-80.

28 Kuo PT, Zeng Z, Salim N, et al. The role of CXCR3 and its chemokine ligands in skin disease and cancer. Front Med 2018;5:271.

29 Nishina S, Yamauchi A, Kawaguchi T, et al. Dipeptidyl peptidase 4 inhibitors reduce hepatocellular carcinoma by activating lymphocyte chemotaxis in mice. Cell Mol Gastroenterol Hepatol 2019;7:115-34.

30 Garrido-Martin EM, Mellows TWP, Clarke J, et al. M1 ${ }^{\text {hot }}$ tumorassociated macrophages boost tissue-resident memory $T$ cells infiltration and survival in human lung cancer. $J$ Immunother Cancer 2020;8:e000778.

31 Zilionis R, Engblom C, Pfirschke C, et al. Single-Cell transcriptomics of human and mouse lung cancers reveals conserved myeloid populations across individuals and species. Immunity 2019;50:1317-34.

32 Zemek RM, De Jong E, Chin WL, et al. Sensitization to immune checkpoint blockade through activation of a STAT1/NK axis in the tumor microenvironment. Sci Transl Med 2019;11:11.

$33 \mathrm{Ma} \mathrm{C}$, Han M, Heinrich B, et al. Gut microbiome-mediated bile acid metabolism regulates liver cancer via NKT cells. Science [Internet].2018:360.

34 Jiang Y, Li Y. Zhu B. T-cell exhaustion in the tumor microenvironment [Internet]. Cell Death \& Disease 2015:e1792.

35 Freeman GJ, Wherry EJ, Ahmed R, et al. Reinvigorating exhausted HIV-specific T cells via PD-1-PD-1 ligand blockade. J Exp Med 2006;203:2223-7.

36 Yost KE, Satpathy AT, Wells DK, et al. Clonal replacement of tumorspecific T cells following PD-1 blockade. Nat Med 2019;25:1251-9.

37 Chen Z, Ji Z, Ngiow SF, et al. TCF-1-Centered Transcriptional Network Drives an Effector versus Exhausted CD8 T Cell-Fate Decision. Immunity 2019;51:840-55.

38 Sade-Feldman M, Yizhak K, Bjorgaard SL, et al. Defining T cell states associated with response to checkpoint immunotherapy in melanoma. Cell 2019:176:404.

39 Desai P, Tahiliani V, Stanfield J, et al. Inflammatory monocytes contribute to the persistence of CXCR ${ }^{\text {hi }}$ CX3CR1 circulating and lung-resident memory $\mathrm{CD} 8^{+} \mathrm{T}$ cells following respiratory virus infection. Immunol Cell Biol 2018;96:370-8.

40 Abboud G, Desai P, Dastmalchi F, et al. Tissue-Specific programming of memory CD8 T cell subsets impacts protection against lethal respiratory virus infection. J Exp Med 2016;213:2897-911.

41 Serbina NV, Salazar-Mather TP, Biron CA, et al. TNF/iNOS-producing dendritic cells mediate innate immune defense against bacterial infection. Immunity 2003;19:59-70.

42 Aldridge JR, Moseley CE, Boltz DA, et al. TNF/iNOS-producing dendritic cells are the necessary evil of lethal influenza virus infection. Proc Natl Acad Sci U S A 2009;106:5306-11.

43 Dunbar PR, Cartwright EK, Wein AN, et al. Pulmonary monocytes interact with effector T cells in the lung tissue to drive TRM differentiation following viral infection. Mucosal Immunol 2020;13:161-71.

44 Chu K-L, Batista NV, Wang KC, et al. GITRL on inflammatory antigen presenting cells in the lung parenchyma provides signal 4 for T-cell 
accumulation and tissue-resident memory T-cell formation. Mucosal Immunol 2019:12:363-77.

45 Desai P, Tahiliani V, Hutchinson TE, et al. The TNF superfamily molecule light promotes the generation of circulating and LungResident memory CD8 T cells following an acute respiratory virus infection. J.i. 2018;200:2894-904.

46 Desai P, Abboud G, Stanfield J, et al. HVEM imprints memory potential on effector CD8 T cells required for protective mucosal immunity. J.i. 2017;199:2968-75.

47 Marigo I, Zilio S, Desantis G, et al. T cell cancer therapy requires CD40-CD4OL activation of tumor necrosis factor and inducible NitricOxide-Synthase-Producing dendritic cells. Cancer Cell 2016;30:651.

48 Pan Y, Tian T, Park CO, et al. Survival of tissue-resident memory T cells requires exogenous lipid uptake and metabolism. Nature 2017:543:252-6.

49 Kastenmüller W, Brandes M, Wang Z, et al. Peripheral prepositioning and local CXCL9 chemokine-mediated guidance orchestrate rapid memory CD8+ T cell responses in the lymph node. Immunity 2013;38:502-13.

50 Kurachi M, Kurachi J, Suenaga F, et al. Chemokine receptor CXCR3 facilitates CD8+ T cell differentiation into short-lived effector cells leading to memory degeneration. J Exp Med 2011;208:1605-20.

51 Groom JR, Richmond J, Murooka TT, et al. Cxcr3 chemokine receptor-ligand interactions in the lymph node optimize CD4+ T helper 1 cell differentiation. Immunity 2012;37:1091-103.

52 Maynard A, McCoach CE, Rotow JK, et al. Therapy-Induced evolution of human lung cancer revealed by single-cell RNA sequencing. Cell 2020;182:1232-51.
53 Perry CJ, Muñoz-Rojas AR, Meeth KM, et al. Myeloid-targeted immunotherapies act in synergy to induce inflammation and antitumor immunity. J Exp Med 2018;215:877-93.

54 Leblond MM, Tillé L, Nassiri S, et al. Cd40 agonist restores the antitumor efficacy of anti-PD1 therapy in muscle-invasive bladder cancer in an IFN I/II-Mediated manner. Cancer Immunology Research 2020;8:1180-92.

55 Hoves S, Ooi C-H, Wolter C, et al. Rapid activation of tumorassociated macrophages boosts preexisting tumor immunity. J Exp Med 2018;215:859-76.

56 Gunderson AJ, Yamazaki T, McCarty K, et al. TGF $\beta$ suppresses CD8+ T cell expression of CXCR3 and tumor trafficking. Nat Commun 2020;11:1749.

57 Etzerodt A, Tsalkitzi K, Maniecki M, et al. Specific targeting of CD163+ TAMs mobilizes inflammatory monocytes and promotes $T$ cell-mediated tumor regression. J Exp Med 2019;216:2394-411.

58 Yokoyama Y, Lew ED, Seelige R, et al. Immuno-oncological efficacy of RXDX-106, a novel TAM (TYRO3, AxI, MER) family small-molecule kinase inhibitor. Cancer Res 2019;79:1996-2008.

59 Molgora M, Esaulova E, Vermi W, et al. Trem2 modulation remodels the tumor myeloid landscape enhancing anti-PD-1 immunotherapy. Cell 2020;182:886-900.

60 Pascual-García M, Bonfill-Teixidor E, Planas-Rigol E, et al. Lif regulates CXCL9 in tumor-associated macrophages and prevents CD8+ T cell tumor-infiltration impairing anti-PD1 therapy. Nat Commun 2019;10:2416. 